\title{
Insulin sensitivity of glucose disposal and lipolysis: no influence of common genetic variants in IRS-1 and CAPN10
}

\author{
M. Stumvoll, H. G. Wahl, F. Machicao, H. Häring \\ Department of Endocrinology and Metabolism, Eberhard-Karls University, Tübingen, Germany
}

\begin{abstract}
Aims/hypothesis. This study aimed to assess the physiologic relationships between insulin sensitivity of lipolysis and that of glucose disposal and whether two common genetic polymorphisms associated with insulin resistance partially explained the remaining variation.

Methods. We measured suppression of lipolysis (isotopically [primed-continuous infusion of d5 glycerol] measured glycerol rate of appearance) and glucose disposal during a three-step $(n=24)$ or standard $(n=$ 57) hyperinsulinaemic euglycaemic clamp in 81 healthy subjects. We also compared insulin sensitivity of lipolysis in carriers (vs. wildtype controls) of the CAPN10 UCSNP43 G/A and IRS-1 Gly972Arg polymorphisms.
\end{abstract}

Results. We observed a significant correlation between insulin sensitivity of glucose disposal and insulin sensitivity of lipolysis $(r=-0.39, p<0.001)$ which was retained, albeit weaker, after adjusting for BMI ( $r=$
$-0.27, p=0.002$ ). No significant difference in insulin sensitivity of lipolysis was found between Gly/Gly compared with $X / A r g$ (IRS-1) and $G / G$ compared with $G / A+A / A(C A P N 10)($ all $p$ values $>0.15)$.

Conclusion/interpretation. Insulin sensitivity of lipolysis has a considerable variation in healthy human beings and independently explains about $10 \%$ of the variation in insulin sensitivity of glucose disposal (or vice versa). It is possible that mediated through NEFAs, insulin resistance of glucose disposal is secondary to that of lipolysis. Alternatively, the biological variation in insulin sensitivity, to some extent, affects both systems in parallel. Neither of the two putatively insulin resistance-related polymorphisms that were tested contributed measurably to the biological variation of insulin sensitivity of lipolysis. [Diabetologia (2002) 45:651-656]

Keywords Insulin resistance, lipolysis, glycerol, stable isotopes, euglycaemic hyperinsulinaemic clamp.
Insulin resistance is a key factor in the development of Type II (non-insulin-dependent) diabetes mellitus. Although the complex pathogenesis of insulin resistance is not completely understood, it is generally accepted that adipose tissue, particularly obesity, is of pivotal

Received: 28 September 2001 / Revised: 14 December 2001

Published online: 4 April 2002

(C) Springer-Verlag 2002

Corresponding author: Dr. M. Stumvoll, Medizinische Universitätsklinik, Otfried-Müller-Str. 10, D-72076 Tübingen, Germany, e-mail: michael.stumvoll@med.uni-tuebingen.de Abbreviations: EC50, Effective concentration causing halfmaximal effect; CAPN1O, calpain-10 gene importance $[1,2]$. It is still not clear, however, whether and which genetic factors are involved [3, 4]. Among the mediators of the cross-talk between adipose tissue and insulin sensitivity, non-esterified fatty acids (NEFAs) are best characterized. They have been shown to influence glucose homeostasis through inhibition of peripheral glucose disposal and stimulation of endogenous glucose production (reviewed in [5]).

Lipolysis, the process by which NEFAs are released from stored triglycerides is highly sensitive to the suppressive effect of insulin [6]. In both Type II diabetes and simple obesity, the suppression of lipolysis is insulin resistant resulting in excessive release of NEFAs into plasma $[7,8,9,10]$. These conditions are 
also invariably associated with insulin resistance of glucose disposal. It is thus possible that insulin resistance of glucose disposal, which ultimately favours the development of Type II diabetes, is a result of insulin resistance of lipolysis. Correlations between insulin sensitivity and NEFA concentrations have been shown [11]. Isotope studies (palmitate, glycerol) in small cohorts provided some indication of a relationship between insulin sensitivity of lipolysis and glucose disposal, although the confounding influence of obesity was not accounted for.

In addition to measuring insulin sensitivity of glucose disposal, to fathom the range and identify extremes in insulin sensitivity of lipolysis in a healthy (predominantly lean) cohort, we did a series of studies using the best available index for systemic lipolysis in vivo: production and release of glycerol into plasma $[12,13]$. Insulin sensitivity of lipolysis was directly measured during a 3-step hyperinsulinaemic euglycaemic clamp $(n=24)$ or estimated from the standard 2-h hyperinsulinaemic clamp $(n=57)$ using a recently validated index derived from the glycerol $\mathrm{Ra}$ at $60 \mathrm{~min}$ [14]. Our first aim was to determine whether and how good a correlation existed between insulin sensitivity of lipolysis and insulin sensitivity of glucose disposal and whether this was independent of obesity (BMI). The second aim was to assess the influence of two common genetic polymorphisms (IRS-1 Gly972Arg and CAPN10 UCSNP43 G/A) on insulin sensitivity specifically of lipolysis. Both variants have been associated previously with insulin resistance $[15,16]$.

\section{Subjects and methods}

Subjects. In the Tübingen Family Study for Type II diabetes more than 700 subjects of Caucasian origin living in the Southwest of Germany have been metabolically characterized to date. As part of a special add-on protocol, lipolysis was determined using stable isotope methodology. At the time of recruitment the genotype was not known and subjects were included in an unselected fashion. We studied 81 healthy, unrelated subjects of whom 24 underwent a 3-step hyperinsulinaemic euglycaemic clamp. Because this procedure is extremely laborious and time consuming, but a much larger number was required for the intended analyses, 57 subjects underwent a standard hyperinsulinaemic euglycaemic clamp. An index of lipolysis from the standard clamp to permit pooling of the data has been validated in a recent paper [14].

The subjects characteristics are shown in Table 1. All subjects underwent the standard preparatory procedures and investigations of the protocol of the Tübingen Family Study (medical history, physical examination, routine blood test, ECG, oral glucose tolerance test). 56 subjects had a positive family history of Type II diabetes (based on a standardized questionnaire). All participants were normal glucose tolerant according to World Health Organisation (WHO) criteria. The protocols were approved by the local ethical committee and after explaining the nature of the study all subjects had given informed written consent.

Experimental protocol. Subjects were studied after an overnight fast. At about $0600 \mathrm{~h}$ an antecubital vein was cannulated for infusion of insulin, glucose and isotopes. A dorsal hand vein on the contralateral arm was cannulated retrogradely and placed under a heating device to permit sampling of arterialized blood. A primed continuous infusion of [2H5]glycerol (Cambridge Isotope Laboratories, Andover, Mass., USA; $1 \mu \mathrm{mol} / \mathrm{kg}, 0.4 \mu \mathrm{mol} / \mathrm{min}$ ) was immediately started. At $0800 \mathrm{~h}$ one of the following clamp protocols was started.

Three-step hyperinsulinaemic-euglycaemic clamp. After the baseline period, subjects received sequential insulin infusions at rates of $0.1,0.25$ and $1.0 \mathrm{mU} \cdot \mathrm{kg}^{-1} \cdot \mathrm{min}^{-1}$ for $2 \mathrm{~h}$ each rate, respectively. Blood was drawn every 5 to $10 \mathrm{~min}$ for determination of blood glucose and a glucose infusion was adjusted appropriately to maintain the baseline glucose concentration. Arterialized blood samples were obtained at $-20,-10$ and 0 min before the start of the insulin infusion and at 100, 110, and 120 min of each 2 -h insulin infusion rate for determination of plasma [2H5]glycerol enrichments, plasma glycerol concentrations and serum insulin and NEFA concentrations.

Table 1. Characteristics of subjects in genotype groups

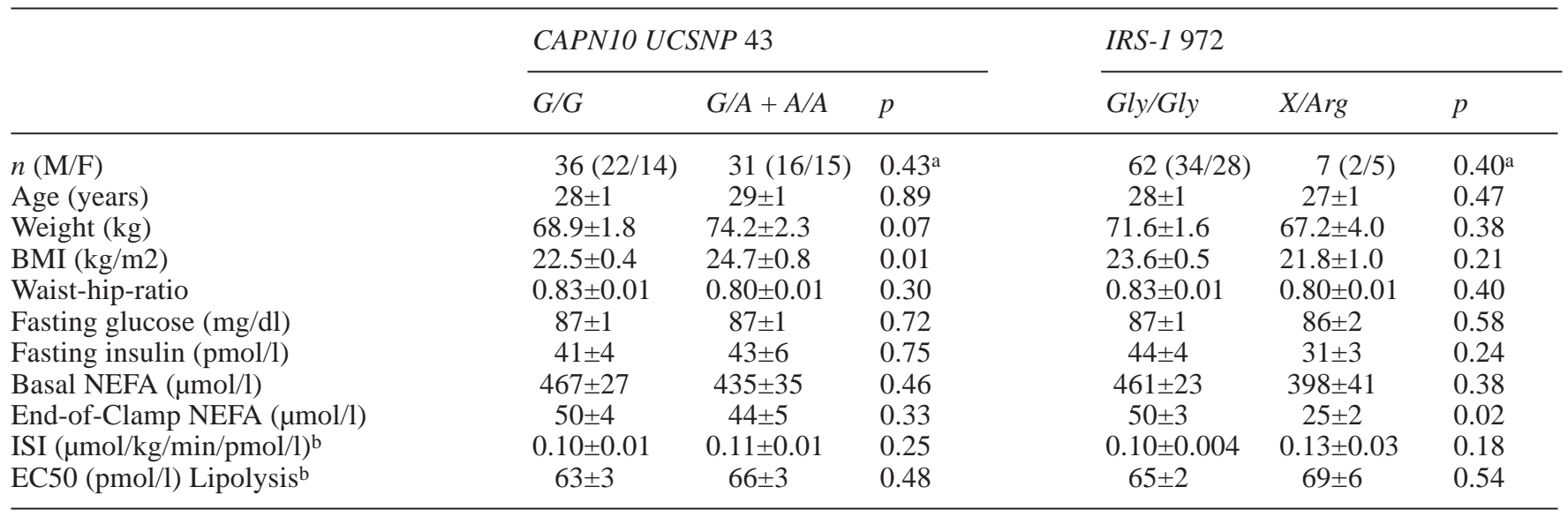

a Chi-squared test

$\mathrm{b}$ adjusted for BMI 
Standard hyperinsulinaemic-euglycaemic clamp. After the baseline period, subjects received a primed insulin infusion at a rate of $1.0 \mathrm{mU} \cdot \mathrm{kg}^{-1} \cdot \mathrm{min}^{-1}$ for $2 \mathrm{~h}$. Blood was drawn every 5 to $10 \mathrm{~min}$ to determine blood glucose and a glucose infusion was adjusted appropriately to maintain the baseline glucose level. Arterialized blood samples were obtained at $-20,-10$ and $0 \mathrm{~min}$ before the start of the insulin infusion and at 30,60, $90,100,110$, and $120 \mathrm{~min}$ to determine plasma [2H5]glycerol enrichments, plasma glycerol concentrations and serum insulin and NEFA concentrations.

Analytical procedures. Blood glucose was determined using a bedside glucose analyser (glucose-oxidase method; YSI, Yellow Springs Instruments, Yellow Springs, Colo., USA). Serum insulin was measured with a microparticle enzyme immunoassay (ABBOTT, Wiesbaden, Germany), serum NEFA with an enzymatic method (WAKO chemicals, Neuss, Germany), plasma glycerol with an enzymatic method (Sigma diagnostics, Deisenhofen, Germany). Plasma [2H5]glycerol enrichment was determined by gas chromatography mass spectrometry using the trimethylsilyl derivative of glycerol. Electron impact ionization was applied and the mass-to-charge ratios 205 and 208 were monitored [17].

Genotyping. The presence of the polymorphism in CAPN10 was determined by sequencing a PCR-amplified product [15], the polymorphism in IRS-1 by PCR and subsequent restriction enzyme analysis with MvaI as previously described [18].

Calculations. The plasma rate of appearance of glycerol (Ra) was used as index for systemic lipolysis. In the steady state, i.e. at baseline and during the last $20 \mathrm{~min}$ glycerol Ra was assumed to equal the rate of disappearance $(\mathrm{Rd})$ and was calculated according to the steady-state equation: $\mathrm{Ra}=\left(\mathrm{ENR}_{\mathrm{inf}} / \mathrm{ENR}_{\mathrm{pl}}-1\right) \cdot \mathrm{F}$, where $\mathrm{ENR}_{\text {inf }}$ is the isotopic enrichment of the infusate, $\mathrm{ENR}_{\mathrm{pl}}$ is the isotopic enrichment of plasma (both in atom \% excess) and $\mathrm{F}$ is the rate of the isotope infusion (in $\mu \mathrm{mol} / \mathrm{min}$ ). The mean of the $-20,-10$ and $0 \mathrm{~min}$ and the 100,110 and $120 \mathrm{~min}$ values of each step were used as steady state values. During the standard hyperinsulinaemic-euglycaemic clamp at 30,60, $90,100,110,120 \mathrm{~min}$, DeBodo's modification of Steele's nonsteady state equations was used [19] to calculate Ra. A fractional pool size of 0.5 and a volume of distribution of $650 \mathrm{ml} / \mathrm{kg}$ were assumed [20].

Insulin sensitivity of systemic lipolysis was assessed as the serum insulin concentration that effectively suppressed plasma glycerol Ra by $50 \%$ of the maximal suppression (EC50). The EC50 for suppression for glycerol Ra from basal rates was estimated by fitting a monoexponential function (Glycerol $\mathrm{Ra}=$ $\max +\mathrm{a} \cdot \mathrm{e}-\mathrm{Ins} \cdot \mathrm{k})$ to the mean basal and the three mean steadystate values where max (= maximal suppression), a and $\mathrm{k}$ represent the fitted parameters and Ins the serum insulin concentration. The EC50 was calculated as EC50 = Ins0 $+\ln 2 / \mathrm{k}$, where Ins0 represents the serum insulin concentration at baseline. In the subjects undergoing the standard hyperinsulinaemic clamp the insulin EC50 for the suppression of lipolysis was estimated using the following equation: EC50=90 minus Glycerol Ra60 min (\% decrease from baseline) times 0.79 . This equation was recently validated to best predict the EC50 in subjects undergoing both a standard and a three-step hyperinsulinaemic euglycaemic clamp [14].

The insulin sensitivity index (ISI, in $\mu \mathrm{mol} \cdot \mathrm{kg}^{-1} \cdot \mathrm{min}^{-1} \cdot \mathrm{pmol} / \mathrm{l}^{-1}$ ) for systemic glucose uptake was calculated as mean infusion rate of exogenous glucose (GIR, in $\mu \mathrm{mol} \cdot \mathrm{kg}^{-1} \cdot \mathrm{min}^{-1}$ ) necessary to maintain euglycaemia during the last $60 \mathrm{~min}$ of the standard clamp (third step of the three-step clamp, respectively) divided by the steady-state serum insulin concentration during step 3.
Statistical analysis. Unless otherwise stated data are given as means \pm SEM. Correlations were tested using linear or nonlinear least square regression analysis. Mathematical adjustment for covariate was done using the residuals from the regression curve. Stepwise multiple linear regression analysis was performed to determine independent correlations with a variable. Statistical comparisons between genotype groups were made using the unpaired Students $t$ test for normally distributed parameters and the non-parametric Wilcoxon rank test for non-normally distributed parameters. Distribution was tested for normality using the Shapiro-Wilk W test. For the purpose of statistical comparison, the insulin EC50 was adjusted for BMI and age. For all analysis a $p$ value of less than 0.05 was considered to be statistically significant. The statistical software package JMP (SAS Institute, Cary, N.C., USA) was used.

\section{Results}

Determinants of insulin sensitivity of glucose disposal and lipolysis. Insulin sensitivity of both glucose disposal and lipolysis were correlated with BMI $(r=0.43$, $p<0.001$ (non-linear) and $r=0.38$ (linear), $p<0.001$, respectively). No other parameter (WHR, age, sex) was independently correlated with insulin sensitivity in our cohort. This indicates that insulin sensitivity of both systems are associated. Interestingly, even after adjusting both insulin sensitivity parameters for the influence of BMI a significant correlation between insulin sensitivity of glucose disposal and that of lipolysis remained (Fig. 1). Also, using stepwise multiple linear regression insulin sensitivity of glucose disposal correlated independently with BMI (entered first, $r=0.40, p<0.001)$ and insulin sensitivity of lipolysis (entered second, $r=0.48, p=0.01$ ). Moreover, fasting NEFA concentrations and insulin sensitivity (ISI) were also highly correlated ( $r=-0.31, p=0.009$, adjusted for BMI). Insulin sensitivity of both lipolysis and glucose disposal varied by as much as $400 \%$ even after adjusting for obesity.

Insulin sensitivity in carriers of polymorphisms in CAPN10 UCSNP43 and IRS-1. Because genetic factors could contribute to this large biologic variation in insulin sensitivity of lipolysis, we tested for an association with common polymorphisms in CAPN1O UCSNP43 and IRS-1. The genotype distributions of the CAPN10 polymorphism (44 G/G, $24 \mathrm{G} / \mathrm{A}, 7 \mathrm{~A} / \mathrm{A}$ ) and the IRS-1 polymorphism (62 Gly/Gly, 7 Gly/Arg, $0 \mathrm{Arg} / \mathrm{Arg}$ ) were in Hardy-Weinberg-equilibrium (all $p>0.50, \chi^{2}$-test).

As shown in Table 1, the groups were reasonably well matched for demographic parameters but neither of the polymorphisms was significantly associated with a difference in insulin sensitivity of either glucose disposal or lipolysis. Only end-of-clamp NEFAs were significantly lower in the X/Arg group compared to the wildtype control group. However, this would indicate a better insulin sensitivity, if any- 

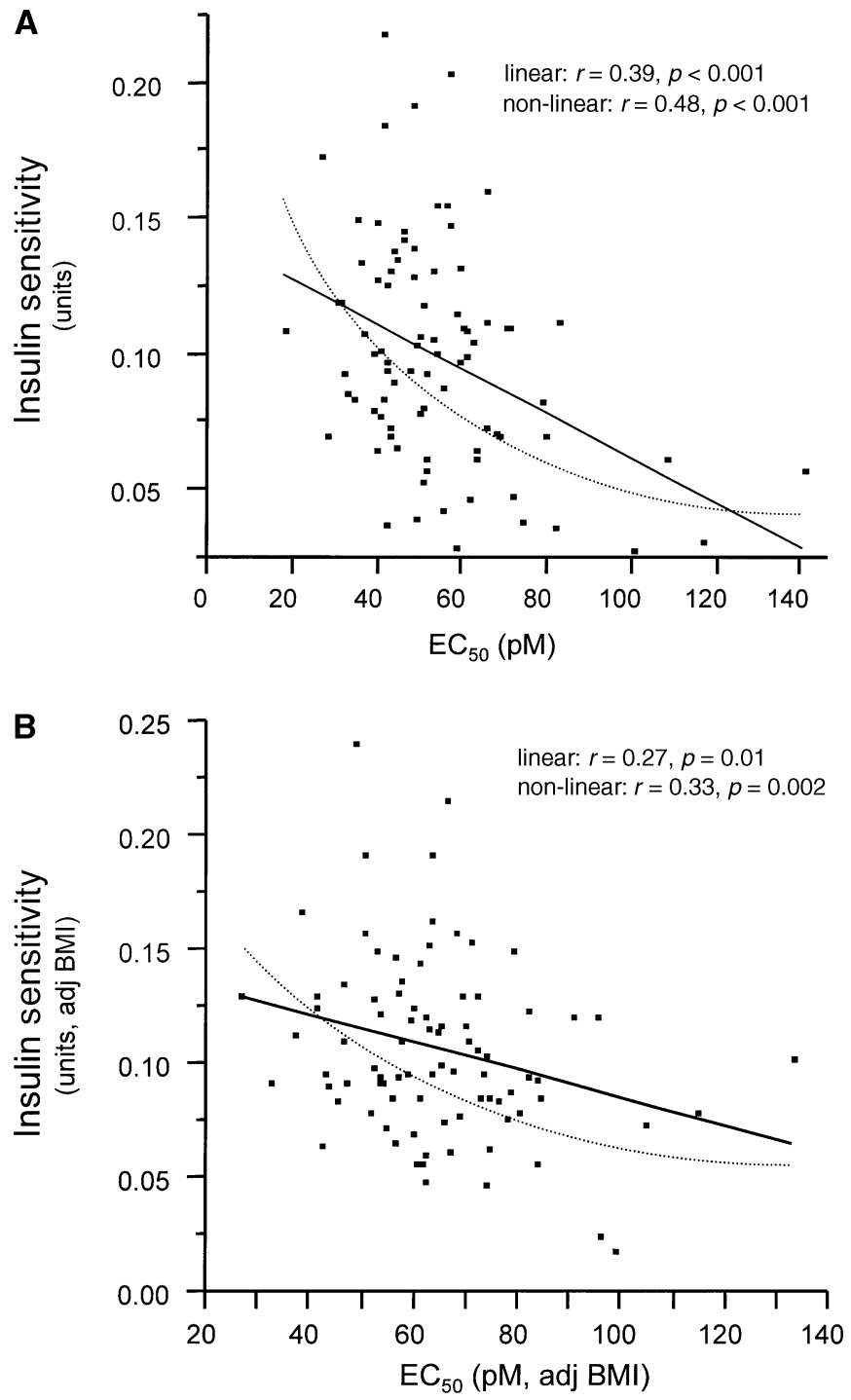

Fig. 1. Correlation between the insulin sensitivity index (glucose disposal for each unit insulin) and suppression of lipolysis (insulin EC50); $\mathbf{A}$ before and $\mathbf{B}$ after adjusting for BMI

thing, in this group. In addition, we compared two "extreme" genotypes. The "high risk" combination IRS-1 (Gly/Arg $)+C A P N 10(G / G)$ had a lower insulin sensitivity of both lipolysis (EC50 $77 \pm 8 \mathrm{pmol} / \mathrm{l}$ ) and glucose disposal $(0.08 \pm 0.02 \mu \mathrm{mol} / \mathrm{kg} / \mathrm{min} / \mathrm{pmol} / \mathrm{l})$ compared to the "low risk" IRS-1 (Gly/Gly) + CAPN10 $(G / A+A / A)$ control group $(67 \pm 3$ pmol/l, $p=0.24 ; 0.11 \pm 0.01 \mu \mathrm{mol} / \mathrm{kg} / \mathrm{min} / \mathrm{pmol} / \mathrm{l}, p=0.29)$. However, due to the small number of subjects $(n=4$ vs. 29) this did not even approach statistical significance (Figs. 2, 3).

Effect of family history of Type II diabetes. In subjects with a family history insulin sensitivity was marginally lower $(0.10 \pm 0.01 \mu \mathrm{mol} / \mathrm{kg} / \mathrm{min} / \mathrm{pmol} / \mathrm{l})$ than in control subjects without family history $(0.116 \pm 0.006 \mu \mathrm{mol} / \mathrm{kg} / \mathrm{min} / \mathrm{pmol} / \mathrm{l}, p=0.10)$. The insulin EC50 for suppression of lipolysis was similar in

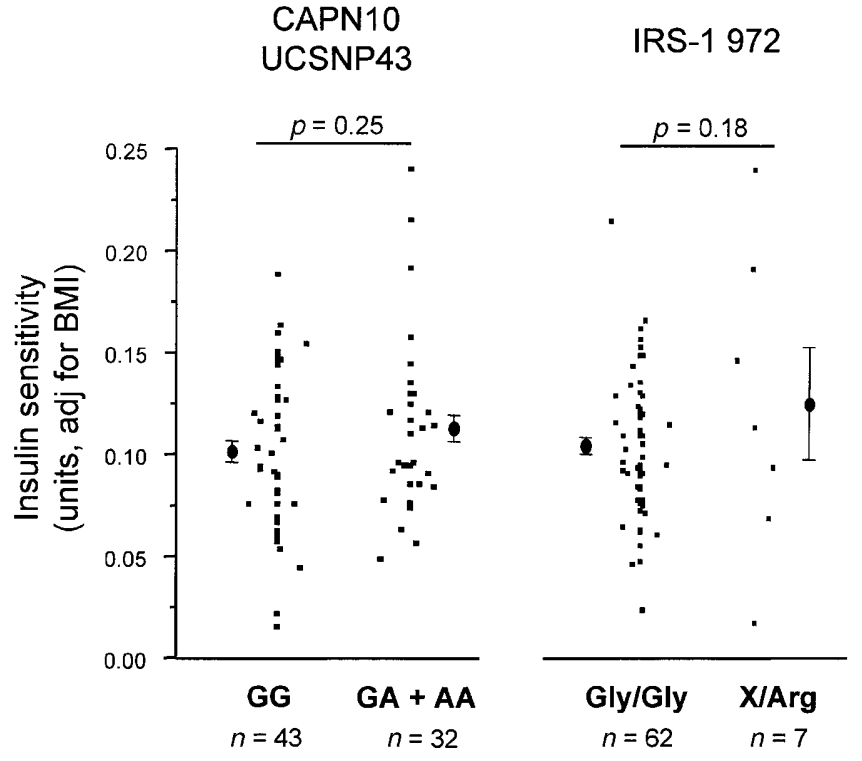

Fig. 2. Insulin sensitivity of glucose disposal in subjects with and without polymorphisms in CAPN10 and IRS-1

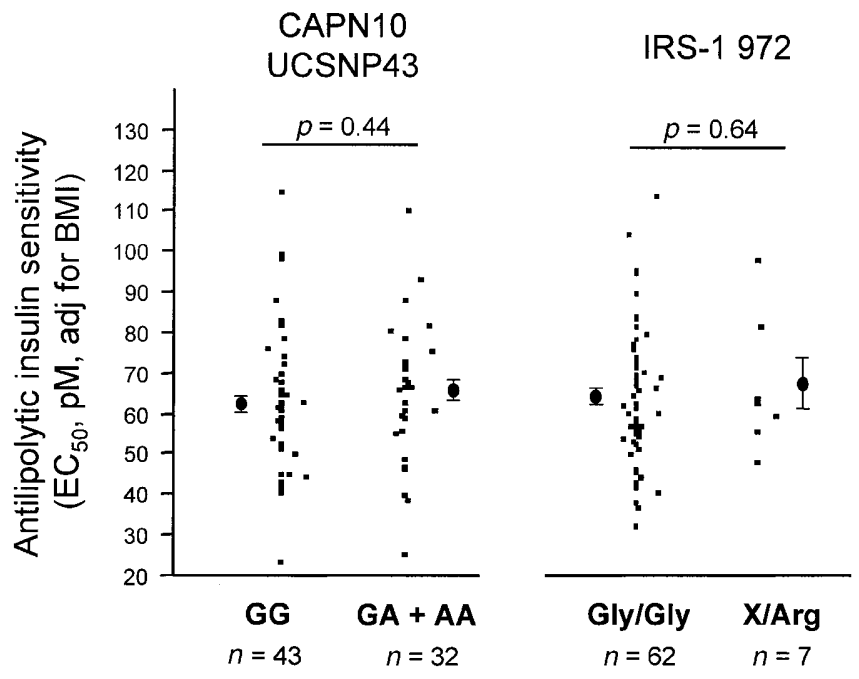

Fig. 3. Insulin sensitivity of suppression of lipolysis (insulin EC50) in subjects with and without polymorphisms in CAPN10 and IRS-1

subjects with a family history $(66 \pm 3 \mathrm{pmol} / \mathrm{l})$ and without $(62 \pm 2 \mathrm{pmol} / \mathrm{l}, p=0.32)$. The frequency of the 2 polymorphisms was not different between subjects with and without family history of Type II diabetes.

\section{Discussion}

This study aimed to assess the relationship between insulin sensitivity of adipose tissue (with respect to insulin's suppressive effect on lipolysis) and glucose disposal. The three-step hyperinsulinaemic clamp which also encompassed the low insulin concentration range (50-100 pmol/l) allowed a very accurate and 
physiologically relevant assessment of insulin sensitivity of lipolysis. The insulin EC50 for suppression of lipolysis in our healthy subjects had an enormous variation and ranged from 20 to 120 pmol/l. A mean EC50 of about $130 \mathrm{pmol} / \mathrm{l}$ was previously reported for insulin resistant, obese subjects [9]. The first major finding of our study was the highly significant correlation between insulin sensitivity of lipolysis and that of glucose uptake which was independent of BMI. Previous studies have shown a correlation between glucose and lipid oxidation in normal [21] and diabetic subjects [10] and also between insulin's glucoregulatory and antilipolytic effect based on NEFA concentrations [22]. However, none of these studies had isotopically determined glycerol release into plasma, or studied a similarly large healthy cohort or accounted appropriately for BMI. Thus, our data present the first evidence that in normal glucose tolerant subjects insulin sensitivity of glucose and NEFA metabolism are (at least statistically) associated.

Our findings might reflect the causal relationship between increased NEFA delivery and muscular insulin sensitivity [5]. Alternatively, because a number of intracellular signalling proteins from the insulin receptor to glucose transport and glycogen synthesis on the one hand and hormone sensitive lipase on the other hand are shared $[23,24]$, the correlation between the two effector systems could reflect the inter-individual variation of the intrinsic level of activity in the common signalling pathway. Regardless of this correlation, however, it is interesting to note that for a given degree of antilipolytic insulin sensitivity that of glucose disposal still varied greatly, and vice versa. This indicates that there may well be a basic, possibly genetically programmed level of insulin sensitivity in both system. But there appears to be plenty of leeway for genetic factors influencing insulin sensitivity of lipolysis and glucose disposal differentially.

Therefore, the logical second aim was to determine whether common genetic polymorphisms previously tested for an association with insulin resistance (CAPN10 UCSNP43 G/A and IRS-1 Gly972Arg) contributed to the remaining variability of insulin sensitivity. In the past, the common Gly972Arg polymorphism in the key insulin signalling protein insulin receptor substrate-1 (IRS-1) represented a prime candidate for a genetic variant contributing to insulin resistance. In vitro studies provided sufficient support for this concept $[25,26]$. The polymorphic allele was found with higher frequency in subjects with Type II diabetes in some [27, 28, 29] but not all studies [30]. Moreover, in a recent study in 333 parent-offspring trios, the $15 \%$ increased risk for Type II diabetes in carriers of the Arg allele failed to reach statistical significance [31]. We had previously failed to find a difference in insulin sensitivity of glucose disposal between carriers of the Arg allele and wildtype controls $[18,32]$. In this study (the study cohort was $100 \%$ dif- ferent from that of our previous report [18]) we also found no significant effect on insulin sensitivity of lipolysis. This supports the notion that the Gly972Arg polymorphism does not contribute to insulin resistance in a major way.

Recently, investigators reported on a single nucleotide polymorphism in intron 3 of the CAPN1O gene (UCSNP-43) the $G$ allele of which appeared to be associated with Type II diabetes [33]. CAPN10 encodes for the cysteine protease calpain-10. Although in diabetic Pima Indians the $G / G$ genotype was not associated with an increased prevalence of Type II diabetes, in normal glucose tolerant Pimas the $G$ allele was associated with decreased glucose disposal strongly suggestive of insulin resistance [15]. In muscle reduced calpain-10 mRNA levels were observed in the $G / G$ genotype [15]. This was thought to be due to mRNA instability before splicing and could result in reduced proteolytic activity. The exact mechanisms, however, as to how this might translate into insulin resistance are far from being understood. Our data showing no difference between the CAPN1O genotypes indicate that the mechanism underlying the reduced insulin sensitivity of the $G$ allele does not involve insulin regulation of lipolysis.

As with any negative finding the question of a type 2 error arises, in our case the probability to erroneously assume no difference between two genotypes when there is in fact a difference. Based on a very similar cohort and using identical methodology, we recently reported an effect of the Prol2Ala polymorphism on insulin sensitivity of lipolysis [12]. The carriers of this polymorphism had approximately $25 \%$ percent lower insulin EC50 compared to the wild type group. In this study the statistical power to detect a similar difference was over $99 \%$. This minimises the risk for a major statistical type 2 error. Nevertheless, the power to detect a $12.5 \%$ difference was only $75 \%$ and to detect a difference of less than $10 \%$ we needed have studied more than 140 subjects. It is of note that the genotype group with the "risk allele" in IRS- 1 and CAPNIO had a $20 \%$ lower insulin sensitivity compared to the group not carrying a "risk allele". It is possible that in a larger cohort such a gene-gene-interaction effect becomes significant.

In conclusion, insulin sensitivity of lipolysis has a considerable variation in healthy humans and is an independent determinant of insulin sensitivity of glucose disposal. It is possible that mediated through NEFAs insulin resistance of glucose disposal is secondary to that of lipolysis. Despite the correlation between insulin sensitivity of lipolysis and glucose disposal there is still a large variability independent of one another, for which genetic factors could be responsible. However, neither of the two common polymorphisms that were tested in this study (CAPN10 UCSNP43 G/A and IRS-1 Gly972Arg) seem to be involved in a major way. 
Acknowledgements. This study was largely supported by the Deutsche Forschungsgemeinschaft DFG (Stu 192/2-1) and in part by a grant from the European Community (QLRT-199900674). We want to thank our laboratory staff for the excellent technical support, in particular A. Wahl, C. Peterfi, E. Maerker and R. Werner.

\section{References}

1. DeFronzo RA, Bonadonna RC, Ferrannini E (1992) Pathogenesis of NIDDM. Diabetes Care 15: 318-368

2. Dinneen S, Gerich J, Rizza R (1992) Carbohydrate metabolism in non-insulin-dependent diabetes mellitus. N Engl J Med 327: 707-713

3. Ferrannini E (1998) Insulin resistance versus insulin deficiency in non-insulin-dependent diabetes mellitus: problems and prospects. Endocr Rev 19: 477-490

4. Gerich JE (1998) The genetic basis of type 2 diabetes mellitus: impaired insulin secretion versus impaired insulin sensitivity. Endocr Rev 19: 491-503

5. Boden G (1997) Role of fatty acids in the pathogenesis of insulin resistance and NIDDM. Diabetes 46: 3-10

6. Stumvoll M, Jacob S (1999) Multiple sites of insulin resistance: muscle, liver and adipose tissue. Exp Clin Endocrinol Diabetes 107: 107-110

7. Nurjhan N, Consoli A, Gerich J (1992) Increased lipolysis and its consequences on gluconeogenesis in noninsulin-dependent diabetes mellitus. J Clin Invest 89: 169-175

8. Campbell PJ, Carlson MG, Nurjhan N (1994) Fat metabolism in human obesity. Am J Physiol 266: E600-E605

9. Groop LC, Bonadonna RC, Simonson DC et al. (1992) Effect of insulin on oxidative and nonoxidative pathways of free fatty acid metabolism in human obesity. Am J Physiol 263: E79-E84

10. Groop LC, Bonadonna RC, DelPrato S et al. (1989) Glucose and free fatty acid metabolism in non-insulin-dependent diabetes mellitus. Evidence for multiple sites of insulin resistance. J Clin Invest 84: 205-213

11. Ferrannini E, Camastra S, Coppack SW et al. (1997) Insulin action and non-esterified fatty acids. The European Group for the Study of Insulin Resistance (EGIR). Proc Nutr Soc 56: 753-761

12. Stumvoll M, Wahl HG, Löblein K et al. (2001) The Pro12Ala polymorphism in the peroxisome proliferator activated receptor $\gamma 2$ gene is associated with increased antilipolytic insulin sensitivity. Diabetes 50: 876-881

13. Stumvoll M, Jacob S, Wahl HG et al. (2000) Suppression of systemic, intramuscular and subcutaneous adipose tissue lipolysis by insulin in humans. J Clin Endocrinol Metab 85: 3740-3745

14. Stumvoll M, Wahl HG, Löblein K et al. (2001) A novel use of the hyperinsulinemic-euglycemic clamp technique to measure insulin sensitivity of systemic lipolysis. (2001) Horm Metab Res 33: 89-95

15. Baier LJ, Permana PA, Yang X et al. (2000) A calpain-10 gene polymorphism is associated with reduced muscle mRNA levels and insulin resistance. J Clin Invest 106: R69-R73

16. Clausen JO, Hansen T, Bjorbaek C et al. (1995) Insulin resistance: interactions between obesity and a common variant of insulin receptor substrate-1. Lancet 346: 397-402
17. Campbell PJ, Carlson MG, Hill JO et al. (1992) Regulation of free fatty acid metabolism by insulin in humans: role of lipolysis and reesterification. Am J Physiol 263: E1063E1069

18. Koch M, Rett K, Volk A et al. (1999) Amino acid polymorphism Gly 972 Arg in IRS-1 is not associated to lower clamp-derived insulin sensitivity in young healthy first degree relatives of patients with type 2 diabetes. Exp Clin Endocrinol Diabetes 107: 318-322

19. DeBodo R, Steele R, Atszuler N et al. (1963) On the hormonal regulation of carbohydrate metabolism: studies with C14 glucose. Recent Prog Horm Res 19: 445-488

20. McCulloch AJ, Johnston DG, Baylis PH et al. (1983) Evidence that thyroid hormones regulate gluconeogenesis from glycerol in man. Clin Endocrinol (Oxf) 19: 67-76

21. Lillioja S, Bogardus C, Mott D et al. (1985) Relationship between insulin-mediated glucose disposal and lipid metabolism in man. J Clin Invest 75: 1106-1115

22. Pei D, Chen Y-D, Hollenbeck CB et al. (1995) Relationship between insulin-mediated glucose disposal by muscle and adipose tissue lipolysis in healthy volunteers. J Clin Endocrinol Metab 80: 3368-3372

23. Eriksson H, Ridderstrale M, Degerman E et al. (1995) Evidence for the key role of the adipocyte cGMP-inhibited cAMP phosphodiesterase in the antilipolytic action of insulin. Biochim Biophys Acta 1266: 101-107

24. Nakanishi H, Brewer KA, Exton JH (1993) Activation of the zeta isozyme of protein kinase $\mathrm{C}$ by phosphatidylinositol 3,4,5-trisphosphate. J Biol Chem 268: 13-16

25. Hribal ML, Federici M, Porzio O et al. (2000) The Gly-Arg972 amino acid polymorphism in insulin receptor substrate-1 affects glucose metabolism in skeletal muscle cells. J Clin Endocrinol Metab 85: 2004-2013

26. Almind K, Inoue G, Pedersen O et al. (1996) A common amino acid polymorphism in insulin receptor substrate-1 causes impaired insulin signaling. Evidence from transfection studies. J Clin Invest 97: 2569-2575

27. Zhang Y, Wat N, Stratton IM et al. (1996) UKPDS 19: heterogeneity in NIDDM: separate contributions of IRS- 1 and beta 3-adrenergic-receptor mutations to insulin resistance and obesity respectively with no evidence for glycogen synthase gene mutations. UK Prospective Diabetes Study. Diabetologia 39: 1505-1511

28. Imai Y, Fusco A, Suzuki Y et al. (1994) Variant sequences of insulin receptor substrate-1 in patients with noninsulindependent diabetes mellitus. J Clin Endocrinol Metab 79: 1655-1658

29. Almind K, Bjorbaek C, Vestergaard H et al. (1993) Aminoacid polymorphisms of insulin receptor substrate-1 in noninsulin-dependent diabetes mellitus. Lancet 342: 828-832

30. Hager J, Zouali H, Velho G et al. (1993) Insulin receptor substrate (IRS-1) gene polymorphisms in French NIDDM families. Lancet 342: 1430-1430

31. Altshuler D, Hirschhorn JN, Klannemark M et al. (2000) The common PPARg Pro12Ala polymorphism is associated with decreased risk of type 2 diabetes. Nat Genet 26: 76-80

32. Stumvoll M, Fritsche A, Volk A et al. (2001) The Gly972Arg polymorphism in the insulin receptor substrate-1 gene contributes to the variation in insulin secretion in normal glucose tolerant humans. Diabetes 50: 882-885

33. Horikawa Y, Oda N, Cox NJ et al. (2000) Genetic variation in the gene encoding calpain-10 is associated with type 2 diabetes mellitus. Nat Genet 26: 163-175 